\title{
Metastasis of breast cancer to an endometrial polyp, the cervix and a leiomyoma: A case report and review of the literature
}

\author{
SULTANA RAZIA ${ }^{1}$, KENTARO NAKAYAMA ${ }^{1}$, MAYU TSUKAO ${ }^{1}$, KOHEI NAKAMURA ${ }^{1}$, MASAKO ISHIKAWA ${ }^{1}$, \\ TOMOKA ISHIBASHI ${ }^{1}$, NORIYOSHI ISHIKAWA ${ }^{2}$, KAORI SANUKI ${ }^{1}$, HITOMI YAMASHITA ${ }^{1}$, RURIKO ONO ${ }^{1}$, \\ MOHAMMAD MAHMUD HOSSAIN ${ }^{1}$, TOSHIKO MINAMOTO ${ }^{1}$ and SATORU KYO $^{1}$
}

Departments of ${ }^{1}$ Obstetrics and Gynecology, and ${ }^{2}$ Organ Pathology, Shimane University School of Medicine, Izumo, Shimane 6938501, Japan

Received July 6, 2016; Accepted July 13, 2017

DOI: $10.3892 / \mathrm{ol} .2017 .6822$

\begin{abstract}
Haematogenous metastases of breast cancer tumors has previously been demonstrated to frequently occur at the sites of the lung, bones, liver and brain, however presence in the uterine remains a rare occurrence. Metastatic carcinoma of the uterus usually originates from other genital sites, most frequently from the ovaries. The current review presents the first reported case of lobular breast carcinoma metastasizing to an endometrial polyp, the cervix and a leiomyoma simultaneously. The patient (58 years, female) first presented with abnormal uterine bleeding. Invasive ductal carcinoma had previously been diagnosed in her right breast, with lobular and ductal cancer cells observed to be present in her lymph nodes. A hysteroscopic procedure to examine the postmenopausal bleeding revealed an endometrial polyp, which was subsequently resected. The morphology and immunohistochemical studies confirmed the diagnosis of metastasis of lobular breast carcinoma to an endometrial polyp. An ${ }^{18} \mathrm{~F}$ fludeoxyglucose positron emission tomography/computed tomography (PET-CT) scan performed following the diagnosis, revealed a slightly increased uptake in the myoma, which is often observed in benign uterine leiomyoma. The patient then underwent a total abdominal hysterectomy with bilateral salpingo-oophorectomy and partial colectomy. Pathology results demonstrated that the uterine leiomyoma and cervix shared the same histopathological features as those presented by the primary lobular breast carcinoma. Although rare, breast tumors may metastasize to an endometrial polyp, cervix and leiomyoma concurrently in patients, therefore physicians may now consider the potential of the diagnosis of metastatic spread to the genital tract, in a patient with abnormal uterine
\end{abstract}

Correspondence to: Dr Kentaro Nakayama, Department of Obstetrics and Gynecology, Shimane University School of Medicine, Enyacho 89-1, Izumo, Shimane 6938501, Japan

E-mail:kn88@med.shimane-u.ac.jp

Key words: metastasis, breast cancer, endometrial polyp, leiomyoma bleeding and a history of lobular breast cancer. Gynecologists planning a laparoscopic hysterectomy for a patient with a history of lobular breast carcinoma may consider abdominal rather than laparoscopic hysterectomy, as lobular carcinoma is difficult to detect. The use of PET-CT may be beneficial for the identification of an unexpected mass.

\section{Introduction}

The female genital tract is rarely involved by metastatic tumors. The most common anatomic locations for metastases to the female genital tract are the ovaries and the vagina. Among extragenital cancers metastasizing to the female genital tract, breast is the most common primary site, followed by the gastrointestinal tract, lung, kidney, and skin (melanoma) $(1,2)$. Metastases to the uterus from extragenital cancer are less common (3), but up to $8 \%$ of breast carcinomas will metastasize to that organ (4). Lobular carcinoma is the most common type of breast carcinoma that metastasizes to the uterus (5). When an extragenital tumor metastasizes to the uterus, it is predominantly located in the myometrium; in a minority of cases, the metastasis is confined to the endometrium $(1,2)$. In this article, we present a case of concurrent metastatic breast carcinoma to an endometrial polyp, a uterine leiomyoma, and the uterine cervix. This is the first reported case with this combination. We also review the literature.

\section{Case report}

A 58-year-old postmenopausal Japanese woman presented to our gynecological outpatient department with abnormal uterine bleeding in 2015. Nine years previously, she had undergone breast-conserving surgery for cancer of the right breast. Histopathologic examination of that mass revealed a stage IIIc invasive ductal carcinoma in the breast, with large groups of lobular cancer cells and very small groups of ductal cancer cells found in the lymph nodes. After surgery of the breast cancer, the patient had been received adjuvant chemotherapy (doxifluridine 5 years, cyclophosphamide 3 years and docetaxel 1 years) radiotherapy (60 Gy to the right breast) and hormone therapy (goserelin acetate 6 years, tamoxifen 4 months, toremifene citrate 3 years). 
A diagnostic work-up was initiated to detect possible causes of vaginal bleeding. She underwent transvaginal ultrasound, which revealed endometrial thickening $(9 \mathrm{~mm})$. Magnetic resonance imaging (MRI) revealed a thickened endometrial myometrium (16x32 mm) with multicystic tumors (Fig. 1). A hysteroscopic examination revealed an endometrial polyp in the uterine cavity, which was resected (Fig. 2). A pathological examination, including hematoxylin-eosin staining and immunohistochemical staining, was performed (Fig. 3). The acquisition of tumor tissues of the patients was approved by the Shimane University Institutional Review Board. Hematoxylin and eosin revealed the absence of solid, alveolar, papillary, or gland-forming units. The tumor cells were arranged in slender linear strands, one to two cells in thickness. The tumor cells may have been dispersed in an irregular fashion in a densely fibrotic stroma. Results of immunohistochemical staining for estrogen receptor, progesterone receptor, and HER2 were positive, whereas those for E-cadherin were negative. The morphology, along with the immunohistochemical findings and the clinical history, supported the diagnosis of lobular breast carcinoma metastatic to the endometrial polyp.

A positron emission tomography/computed tomography (PET-CT) examination showed slightly increased uptake in the myoma. The standardized uptake value (SUV) on the PET was 9 in the early phase and 11 in the late phage. Multiple reports suggest that mild or moderate uptake of ${ }^{18} \mathrm{~F}$-fludeoxyglucose is often observed in benign uterine leiomyoma and should not be confused with malignant accumulation, and our experience is consistent with this observation. Therefore, the tumor was assumed to be benign, and a laparoscopic hysterectomy was planned. However, because of the large size of the uterus and copious adhesions between the ovary, uterus, and rectum, the procedure was changed to an abdominal hysterectomy. The patient underwent total abdominal hysterectomy, bilateral salpingo-oophorectomy, and partial colectomy. Interestingly, the uterine leiomyoma and cervix shared the same histopathologic features as the primary lobular carcinoma of the breast, indicating that metastatic tumor arose from the original breast carcinoma after a 9-year interval. At the time of this report, the patient is alive and receiving aromatase inhibitor therapy.

\section{Discussion}

Metastases to the female genital tract from extragenital cancers are less common than metastases from genital tract cancers and most often affect the ovaries, with the breast and gastrointestinal tract being the most common sites of the primary tumor (2). The ovaries are often first in the path of spread of malignant cells. They provide an optimal environment for implantation of malignant cells, because they are very vascular with a well-developed lymph network. Additionally, the stroma of ovaries has a favorable $\mathrm{pH}$ and oxygen pressure (6). On the other hand, the other female genital tract organs, including the uterus, seem resistant to metastasis. Mazur et al (1) reported that, among 149 metastatic tumors to the female genital tract from extragenital primaries, the ovary and vagina were the most frequent locations of metastases (75.8 and $13.4 \%$, respectively), while only $8.1 \%$ were to the uterus $(4.7 \%$ to the endometrium, $3.4 \%$ to the cervix). Breast was the second-most

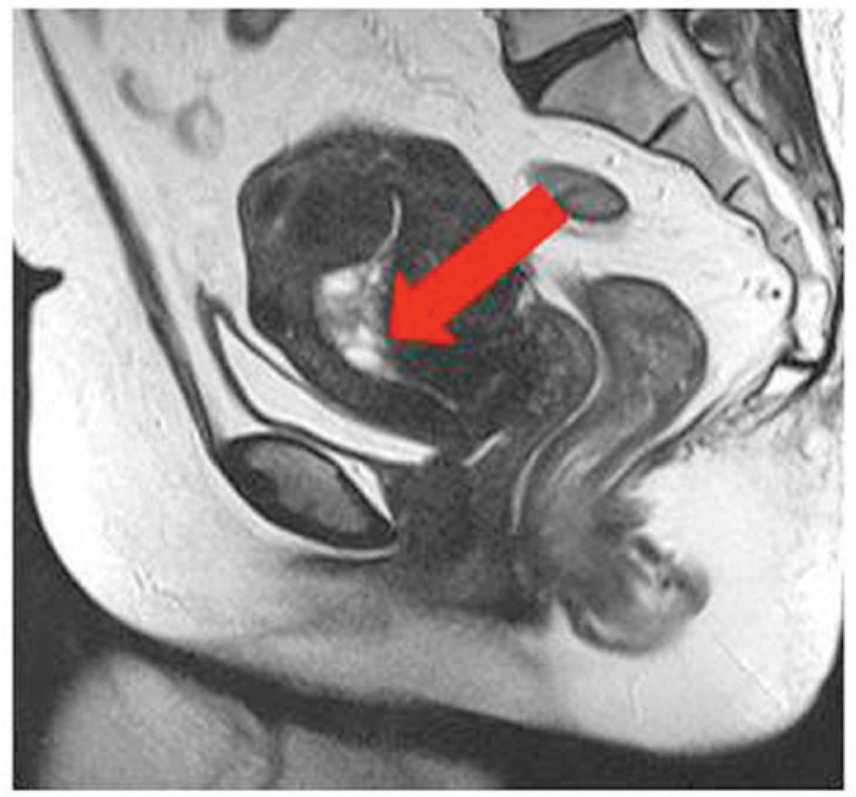

Figure 1. Magnetic resonance imaging of the pelvis. T2-weighted imaging revealed thickened endometrial myometrium $(16 \times 32 \mathrm{~mm})$ with multicystic tumors.

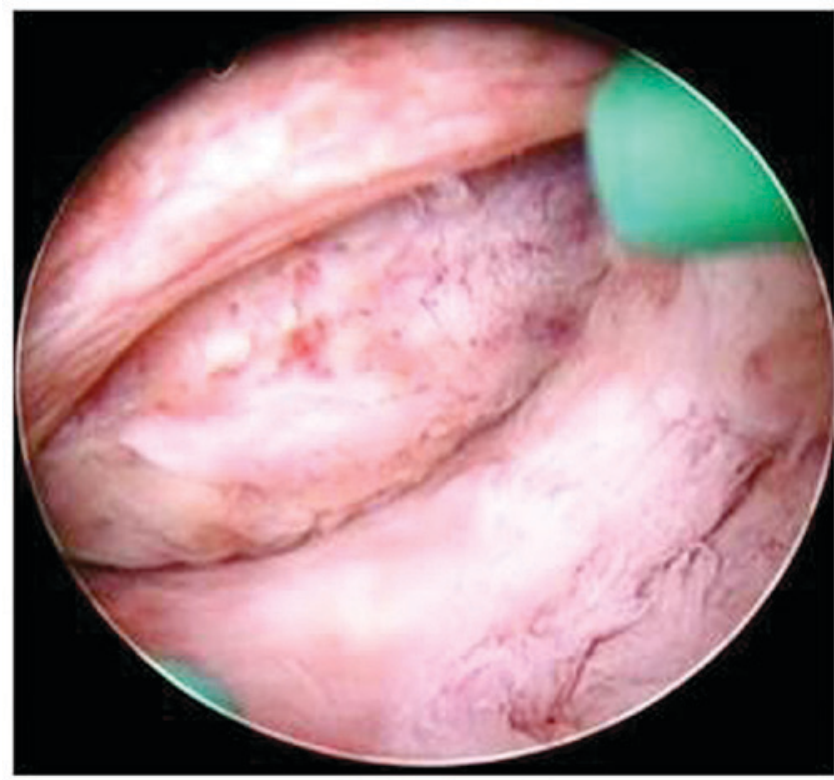

Figure 2. Polyp-like tumor in the uterine cavity. The surface of the polyp is smooth.

common primary site after gastrointestinal tumors; of 52 breast cancer cases metastatic to the gynecologic organs, ovaries were affected in $88.5 \%$ cases, vagina in $5.8 \%$, endometrium in $3.8 \%$, vulva in $1.9 \%$ and none to the cervix (1).

Metastases to the uterus from extragenital cancers are significantly rarer than metastases to the ovaries. Piura et al (7) reported 198 cases of extragenital metastases to the uterus, 112 of which originated from primary breast carcinoma. However, reports of breast cancer metastasis to uterine leiomyoma were limited. Leiomyoma of the uterus is the most common benign tumor in women, and it can be found in $20 \%$ of women at autopsy (8). The low incidence of cancer 

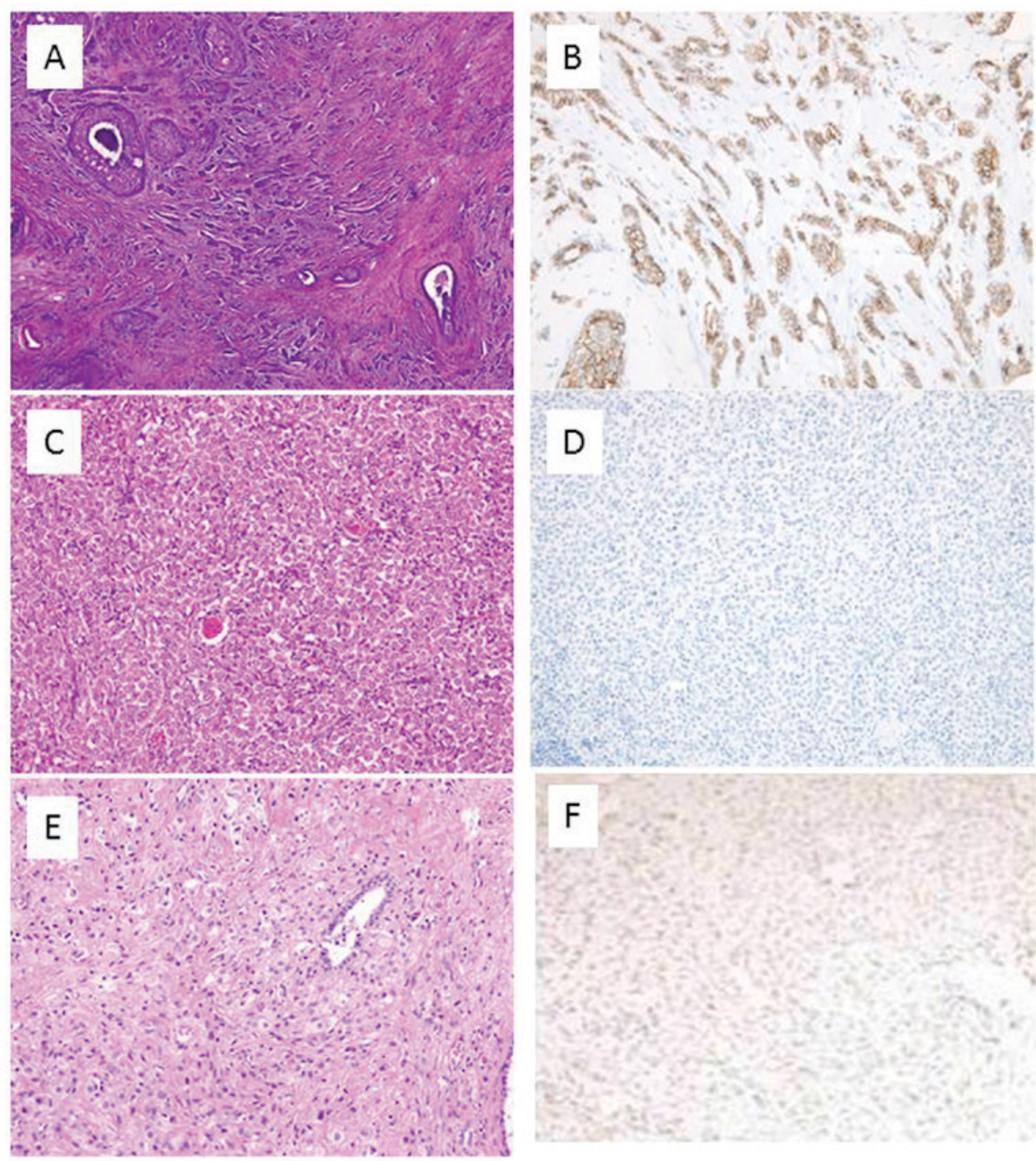

Figure 3. Pathological examination. (A) microscopic image of ductal carcinoma of the right breast. (B) Immunohistochemical staining positive for E-cadherin of ductal carcinoma. (C) Microscopic image of lobular carcinoma of the lymph node. (D) Immunohistochemical staining negative for E-cadherin of lobular carcinoma of lymph node. (E) Metastatic tumor cells from breast lobular carcinoma of leiomyoma, cervix and an endometrial polyp (hematoxylin and eosin). (F) Immunohistochemical staining negative for E-cadherine of uterus.

metastatic to uterine leiomyoma may be explained by the lack of routine autopsy. Kondo et al (9) described presenting signs and symptoms that may result from metastasis to a uterine leiomyoma in 13 cases, including abnormal bleeding in 6 cases $(6 / 13,46.2 \%)$, enlargement of the uterus in 4 cases, and no symptoms in 3 cases. In view of these observations, abnormal uterine bleeding may be a characteristic of a patient with metastasis to a uterine leiomyoma. Our review of the literature regarding metastasis of a carcinoma to a uterine leiomyoma is summarized in Table I. Reviewing the histological types of breast cancer in these reports, ductal carcinoma comprised $61.55 \%(8 / 13)$ and lobular carcinoma comprised $38.4 \%(5 / 13)$ of metastases to uterine leiomyoma. In our case, the patient was diagnosed with both ductal and lobular breast carcinoma, but only lobular carcinoma metastasized to the uterine leiomyoma, and abnormal uterine bleeding was the first sign of metastasis.

Most extragenital metastases to the cervix arise from primary tumors of the gastrointestinal tract (1). Other primary sites include the lung, pancreas, melanoma, urethra, and breast. Breast cancer often metastasizes to the ovary, but rarely to the cervix. Possible explanations for this rarity include the small size of the cervix, its reduced blood flow and distal circulation, and the presence of abundant fibrous tissue, which make the uterine cervix unfavorable for the propagation of malignant cells (10). Yazigi et al (11) summarized the first 24 cases in the literature of breast metastasis to the uterine cervix from his series and previous authors in 1988. Two subsequent cases were reported by Piura et al (7), and Kennebeck and Alagoz (12). Yazigi drew some general conclusions of the patients reviewed. More than two-thirds had vaginal bleeding as a presenting symptom, and greater than $60 \%$ had no evidence of disease on examination. Thus, the metastasis would have been missed if complete evaluations had not been performed, including Pap smear, colposcopy, and biopsy. In our case, the patient had irregular vaginal bleeding, and her cervical Pap smear was normal. In most cases, cervical metastasis of breast cancer is a manifestation of widespread disease. In $67-89 \%$ of the reported cases, distant metastases of other sites were present at diagnosis 


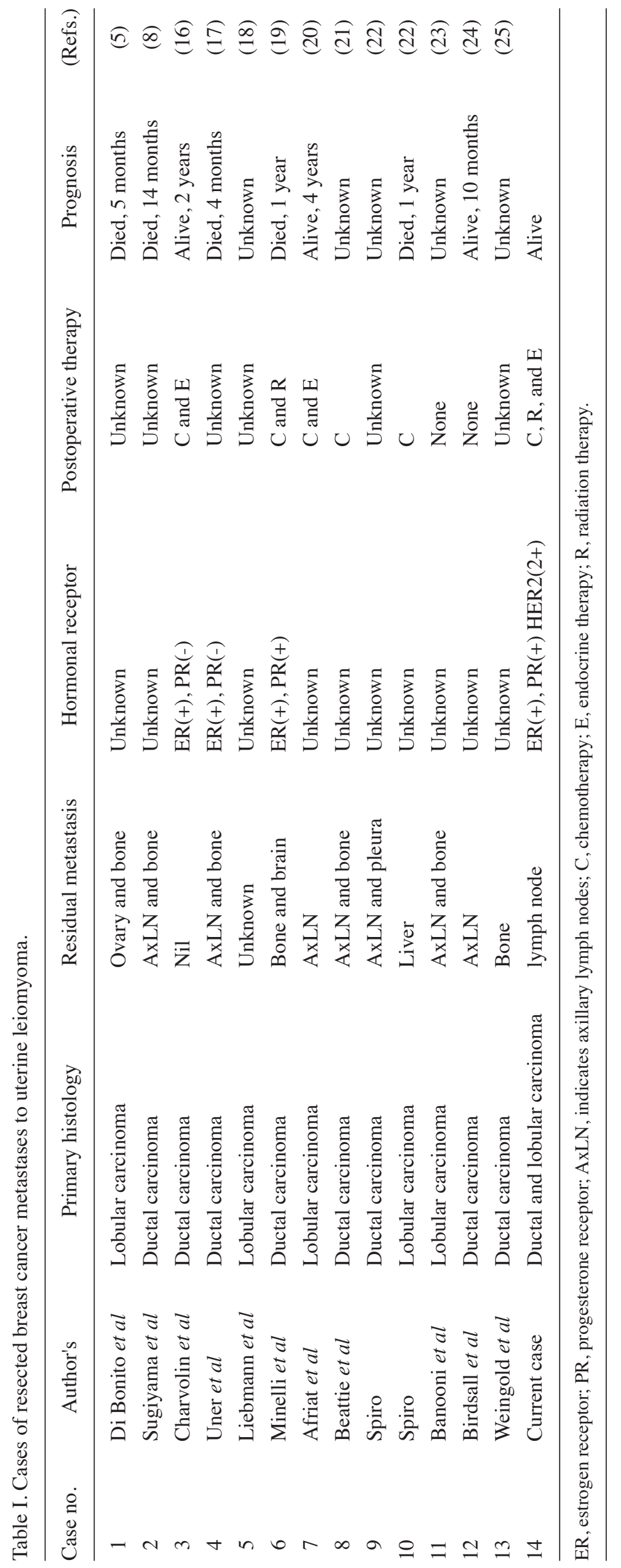




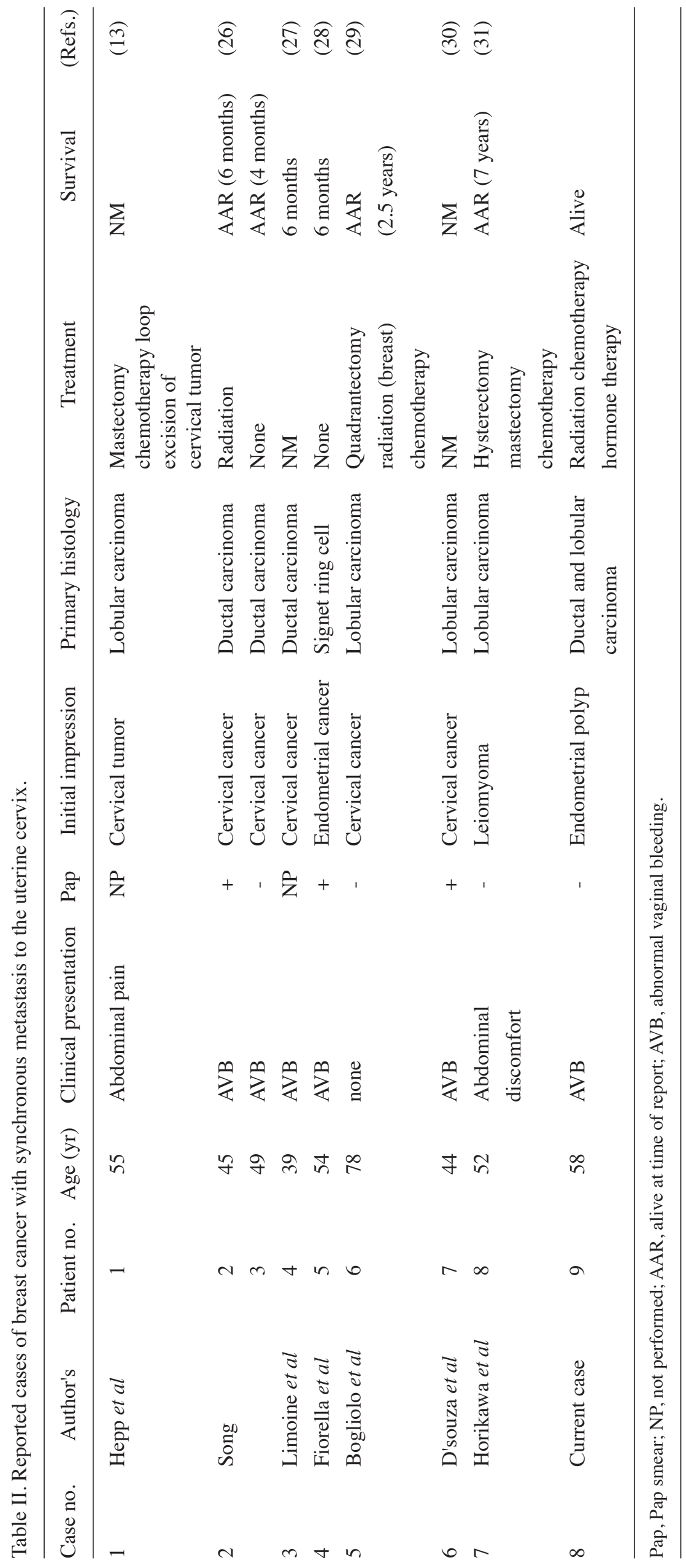




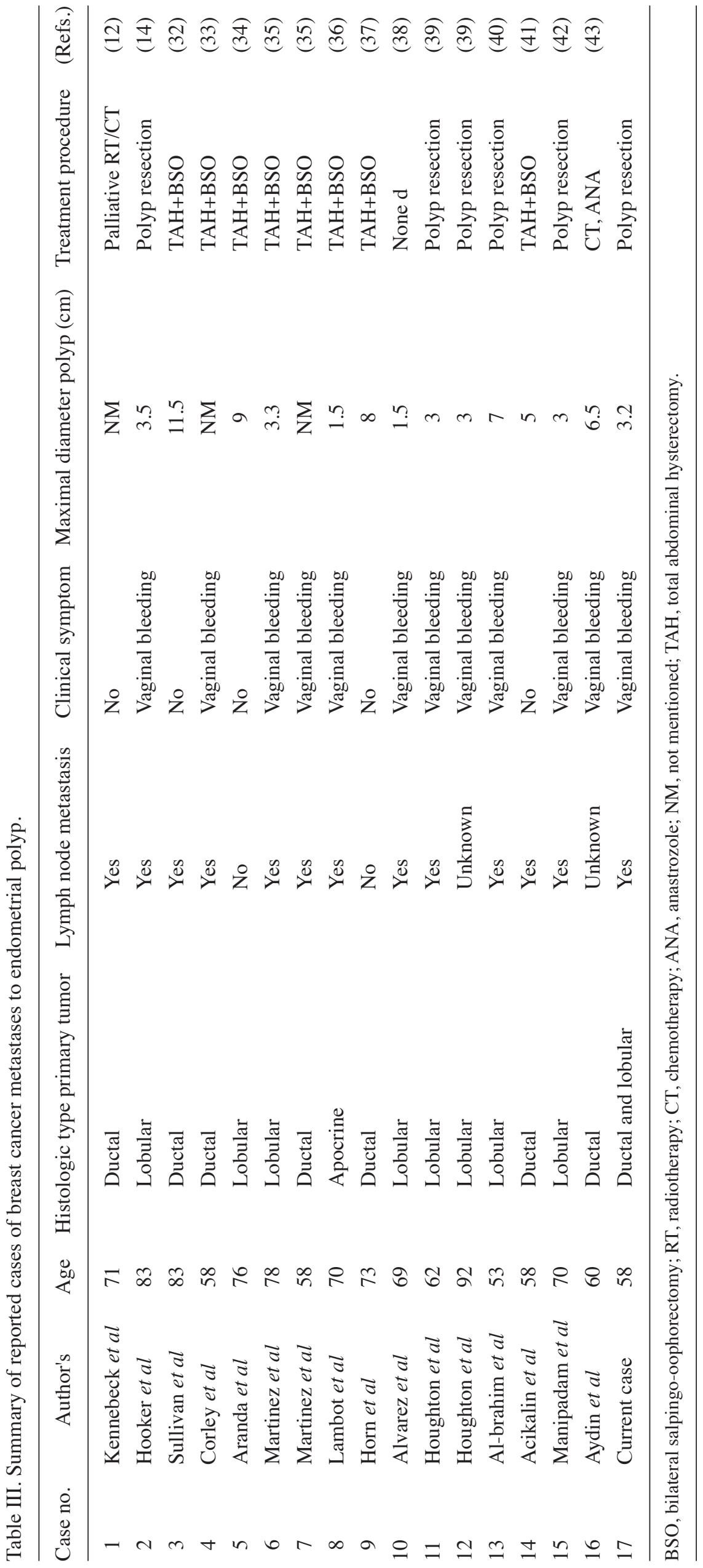


of cervical metastasis $(12,13)$. The response of the cervix to metastatic disease is fibrous proliferation and an inflammatory cellular reaction, which may explain the clinical finding of an expanded, indurated cervix (2). After reviewing the literature, 8 cases (including our case) of metastatic breast carcinoma to the cervix are presented in Table II. Of these 8 cases, $3(37.5 \%)$ had ductal carcinoma and $5(62.5 \%)$ had lobular carcinoma.

Endometrial metastasis from a breast cancer is also uncommon. Kumar and Hart (2) found 2 of 63 cases (3.8\%) and Mazur et al (1) found 7 of 149 cases (4.7\%) with metastases to the endometrium. Polyps are the most common benign lesion in the endometrium, and metastasis to a polyp is exceptional. Abrams et al (4) described 15 cases of breast cancer metastatic to endometrial polyps. Of them, 7 patients had lobular breast cancer, 7 had ductal adenocarcinoma, and 1 had an apocrine type. Ductal carcinoma accounts for approximately $70-75 \%$ of all breast cancers, while lobular carcinoma only accounts for 5-20\%. Despite its lower incidence among breast cancers, lobular carcinoma is the most frequent histologic type that metastasizes to the female genital tract, being found in more than $80 \%$ of all cases. In our case, the patient was diagnosed invasive ductal carcinoma in the breast; lobular and ductal carcinoma both were found together in the lymph node, but histological examination of the endometrial polyp identified only metastatic lobular carcinoma. Thus, our study supports the observation that the metastatic potential of lobular carcinoma for gynecologic organs is higher than that of ductal carcinoma. After analyzing 261 cases of breast cancer, Lamovec and Bracko concluded that lobular carcinoma spreads more frequently to gynecologic organs than ductal carcinoma does (15). This may be explained by the more frequent loss of the adhesive molecule E-cadherin in lobular carcinoma. We believe that a similar phenomenon might have taken place in our patient. A summary of all reported cases to date with metastatic carcinoma to an endometrial polyp is presented in Table III.

Uterine metastases from extragenital cancers are significantly rarer than metastases to the ovary, and when they occur, they are usually secondary to lymphatic spread from preceding ovarian metastases. Metastases restricted to the uterus, without involvement of the ovaries, are indeed very rare and can be explained by hematogenous spread.

In our case, lobular breast carcinoma metastasized to a uterine leiomyoma, the cervix, and an endometrial polyp concurrently, which is exceedingly rare. Although the ovary and vagina are the most common locations of metastasis, the entire organ system is at risk for metastatic disease. Therefore, abnormal uterine bleeding in patients with a history of breast carcinoma should always alert the physician to consider the diagnosis of metastatic spread to the genital tract. Gynecologists who are planning a laparoscopic operation for the patient with a history of lobular breast cancer should consider abdominal dissection rather than laparoscopy, even if the tumor is thought to be benign. If lobular carcinoma remains dormant in the myoma or other gynecological organs, as our case, and the surgeon misses any tissue during the operation, residual disease may spread elsewhere in the peritoneal cavity. Before surgery, the use of PET-CT may be beneficial for identifying an unanticipated mass.

\section{References}

1. Mazur MT, Hsuesh S and Gersell DJ: Metastases to the female genital tract. Analysis of 325 cases. Cancer 53: 1978-1984, 1984.

2. Kumar NB and Hart WR: Metastases to the uterine corpus from extragenital cancer. A clinicopathological study of 63 cases. Cancer 50: 2163-2169, 1982.

3. Charache H: Metastatic carcinoma in the uterus. AM J Surg 53: 152-157, 1941.

4. Abrams HL, Spiro R and Goldstein N: Metastases in carcinoma: Analysis of 1000 autopside cases. Cancer 3: 74-85, 1950.

5. Di Bonito L, Patriarca S and Alberico S: Breast carcinoma metastasizing to the uterus. Eur J Gynaecol Oncol 6: 211-217, 1985.

6. Perisić D, Jancić S, Kalinović D and Cekerevac M: Metastasis of lobular breast carcinoma to the cervix. J Obstet Gynaecol Res 33: 578-580, 2007.

7. Piura B, Yanai-Inbar I, Rabinovich A, Zalmanov S and Goldstein J: Abnormal uterine bleeding as a presenting sign of metastases to the uterine corpus, cervix and vagina in a breast cancer patient on tamoxifen therapy. Eur J Obstet Gynecol Reprod Biol 83: 57-61, 1999.

8. Sugiyama T, Toyoda N, Nose J, Kihira N, Ando Y and Ishihara A: Breast cancer metastatic to uterine leiomyoma: A case report. J Obstet Gynaecol (Tokyo 1995) 21: 349-355, 1995.

9. Kondo NI, Yoshida S, Kajiyama H, Nagasaka T and Uematsu T: Metastasis of breast cancer to a uterine leiomyoma. Breast Cancer 16: 157-161, 2009.

10. Pérez-Montiel D, Serrano-Olvera A, Salazar LC, Cetina-Pérez L, Candelaria M, Coronel J, Montalvo LA and de León DC: Adenocarcinoma metastatic to the uterine cervix: A case series. J Obstet Gynaecol Res 38: 541-549, 2012.

11. Yazigi R, Sandstad R and Munoz A: Breast cancer metastasizing to the uterine cervix. Cancer 61: 2558-2560, 1988.

12. Kennebeck $C$ and Alagoz T: Signet ring breast carcinoma metastases limited to the endometrium and cervix. Gynecol Oncol 71: 461-464, 1998.

13. Hepp HH, Hoos A, Leppien G and Wallwiener D: Breast cancer metastatic to the uterine cervix: Analysis of a rare event. Cancer Invest 17: 468-473, 1999.

14. Hooker AB, Radder CM, van de Wiel B and Geenen MM: Metastasis from breast cancer to an endometrial polyp; treatment options and follow-up. Report of a case and review of the literature. Eur J Gynaec Oncol 32: 228-230, 2011.

15. Lamovec J and Bracko M: Metastatic pattern of infiltrating lobular carcinoma of the breast: An autopsy study. J Surg Oncol 48: 28-33, 1991

16. Charvolin JY, Salmon RJ, Pecking A and Mareschal V: Positron emission tomography detection of breast cancer metastasis to the uterus. Obstet Gynecol 99: 915-917, 2002.

17. Uner A, Tiras MB, Kilic D, Dursun A and Dilek U: Uterine lipoleiomyoma containing metastatic breast carcinoma: A case with two unusual pathologies. Eur J Obstet Gynecol Reprod Biol 106: 76-78, 2003.

18. Liebmann RD, Jones KD, Hamid R and Lapsley M: Fortuitous diagnosis in a uterine leiomyoma of metastatic lobular carcinoma of the breast. Histopathology 32: 577-578, 1998.

19. Minelli L, Romagnolo C, Giambanco L and Bongiorno E: Uterine leiomyoma metastasis as a first sign of breast cancer. J Am Assoc Gynecol Laparosc 5: 213-215, 1998.

20. Afriat R, Lenain H, Vuagnat C, Michenet P, Luthier F, Maitre F and Grossetti D: Metastasis of breast cancer to a uterine leiomyoma. J Gynecol Obstet Biol Rep (Paris) 22: 243-244, 1993 (In French).

21. Beattie GJ, Duncan AJ, Paterson AJ, Williams AR and Geirsson RT: Breast carcinoma metastatic to uterine leiomyoma. Gynecol Oncol 51: 255-257, 1993.

22. Spiro RK: Breast cancer metastatic to uterine leiomyoma. J Med Soc N J 76: 285-287, 1979.

23. Banooni F, Labes J and Goodman PA: Uterine leiomyoma containing metastatic breast carcinoma. Am J Obstet Gynecol 111: 427-430, 1971.

24. Birdsall CJ, Dockerty MB and Pratt JH: Mammary carcinoma metastasis to uterine myoma. Obstet Gynecol 23: 229-231, 1964.

25. Weingold $\mathrm{AB}$ and Boltuch SM: Extragenital metastases to the uterus. Am J Obstet Gynecol 82: 1267-1272, 1961.

26. Song J: Metastatic carcinoma of the uterine cervix from primary breast cancer. JAMA 184: 498-500, 1963.

27. Limoine NR and Hall PA: Epithelial tumors metastatic to the uterine cervix. A study of 33 cases and review of the literature. Cancer 57: 2002-2005, 1986. 
28. Fiorella RM, Beckwith LG, Miller LK and Kragel PJ: Metastatic signet ring carcinoma of the breast as a source of positive cervicovaginal cytology. A case report. Acta Cytol 37: 948-952, 1993.

29. Bogliolo S, Morotti M, Valenzano Menada M, Fulcheri E, Musizzano Y and Casabona F: Breast cancer with synchronous massive metastasis in the uterine cervix: A case report and review of the literature. Arch Gynecol Obstet 281: 769-773, 2010.

30. D'souza MM, Sharma RS, Tripathi M, Saw SK, Anad A, Singh D and Mondal A: Cervical and uterine metastasis from carcinoma of breast diagnosed by PET/CT. An unusual presentation. Clin Nucl Med 35: 820-823, 2010

31. Horikawa M, Mori Y, Nagai S, Tanaka S, Saito S and Okamoto T: Metastatic breast cancer to the uterine cervix mimicking a giant cervical leiomyoma. J Med Sci 74: 347-351, 2012.

32. Sullivan LG, Sullivan JL and Fairey WF: Breast carcinoma metastatic to endometrial polyps. Gynecol Oncol 39: 96-98, 1990.

33. Corley D, Rowe J, Curtis MT, Hogan WM, Noumoff JS and Livolsi VA: Postmenopausal bleeding from unusual endometrial polyps in women on chronic tamoxifen therapy. Obstet Gynecol 79: 111-116, 1992.

34. Aranda FI, Laforga JB and Martinez MA: Metastasis from breast lobular carcinoma to an endometrial polyps: Reports of a case with immunohistochemical study. Acta Obstet Gynecol Scand 72: 585-587, 1993

35. Martinez-Montero I, Dominguez-Cunchillos F, Muruzabal JC, de Míguel C, Recari E and Ezcurdia M: Uterine metastases from breast cancer. Acta Obstet Gynecol Scand 78: 165-167, 1999.

36. Lambot MA, Eddafali B, Simon P, Fayt I and Noël JC: Metastasis from apocrine carcinoma of the breast to an endometrial polyp. Virchows Arch 438: 517-518, 2001.
37. Horn LC, Einenkel $\mathrm{J}$ and Baier D: Endometrial metastasis from breast cancer in a patient receiving tamoxifen therapy. Gynecol Obstet Invest 50: 136-138, 2000.

38. Alvarez C, Ortiz-Rey JA, Estévez F, Estevez F and de la Fuente A: Metastatic lobular breast carcinoma to an endometrial polyps diagnosed by hysteroscopic biopsy. Obstet Gynecol 102: 1149-1151, 2003.

39. Houghton JP, Ioffe OB, Silverberg SG, McGrady B and McCluggaga WG: Metastatic breast lobular carcinoma involving tamoxifen-associated endometrial polyps: Report of two cases and review of tamoxifen-associated polypoid uterine lesions. Mod Pathol 16: 395-398, 2003.

40. Al-brahim N and Elavathil LJ: Metastatic breast lobular carcinoma to tamoxifen-associated endometrial polyp: Case report and literature review. Ann Diagn Pathol 9: 166-168, 2005.

41. Acikalin MF, Oner U, Tekin B, Yakuz E and Cengiz O: Metastastasis from breast carcinoma to a tamoxifen-related endometrial polyp. Gynecol Oncol 97: 946-948, 2005

42. Manipadam MT, Walter NM and Selvamnani B: Lobular carcinoma metastasis to endometrial polyp unrelated to tamoxifen. Report of a case and review of the literature. APMIS 116: 538-540, 2008.

43. Aydin O, Bagic P, Akyildiz EU, Ozguroglu M and Ilvan S: Metastasis from breast carcinoma to endometrial polyp. Eur J Gynaecol Oncol 29: 666-668, 2008. 